\title{
Digital photogrammetry of historical aerial photographs using open-source software
}

\author{
José Ramón Martínez Batlle ${ }^{1}$ \\ 1 Autonomous University of Santo Domingo (UASD), joseramon@geografiafisica.org
}

\begin{abstract}
Several collections of aerial photographs have been acquired in the Dominican Republic during the last 70 years. Although many of these sources are increasingly becoming available as scanned images, limited digital photogrammetric processing has been done, mainly because of the unaffordable prices of proprietary software licenses and the lack of clear workflows for processing historical photos. Many of the software tools developed in the last two decades, designated as structure-from-motion and multi-view stereo techniques, are bundled within OpenDroneMap, which is an open-source toolkit originally designed for processing aerial drone imagery. The aim of this research was to test the capabilities of this toolkit for the production of accurate orthomosaics, point clouds, and digital surface models from historical aerial photos. A workflow was designed and tested with 10 color aerial photos taken in 2003 of the Ocoa River system, which is located in the southern part of the Dominican Republic. The workflow comprised the preparation of images, selection of ground control points, reconstruction or bundle-block adjustment, dense matching with two different algorithms, and finally the generation of digital surface models (DSMs) and orthomosaics. The results showed that both the orthomosaics and DSMs showed good agreement with the reference data. The main conclusion was that the proposed workflow may be applicable to other historical aerial photographs, with high efficiency and at zero cost.
\end{abstract}

\section{Introduction}

In the Dominican Republic (DR), several collections of historical aerial photos have been preserved by government institutions. These collections are a valuable record of the changes that have occurred in the country during previous decades. The most recent and comprehensive survey, which photographed the DR between 2000 and 2003, was called "INDRHI flight" (color photos, 1:20000 scale). This acronym refers to the National Institute for Hydraulic Resources [29] (see Figure 1). Other nationwide surveys have been labeled MARENA, DR-A/DR-B, and ICM-58 25 27]. Although an increasing number of these photos are becoming available as scanned images, limited digital photogrammetric processing has been done on them.

Recently, the emergence and availability of high-quality digital consumer cameras, mounted as payloads in unmanned aerial systems (hereafter UASs), have fostered modern photogrammetric methods for 3D reconstructions 43. Many software tools, which originated in the computer vision field and can be referred to as structure-from-motion (SFM) and multi-view stereo (MVS) techniques, have been developed to perform complete photogrammetric workflows 1, 17, 48].

Several commercial software packages are available on the market for photogrammetric production, including Acute3D, Pix4D, Agisoft PhotoScan, and ERDAS IMAGINE, which offer complete GUIs and online assistance. On the other hand, open-source software packages offer low-budget and accurate solutions, with the main ones being OpenDroneMap, MicMac, E-foto, Mapillary, and BundlerTools $3,12,31,32,44$.

OpenDroneMap (hereafter ODM) is an open-source toolchain written in the Python programming language. It can be used to perform photogrammetric workflows and was originally designed for processing UAS imagery [3]. ODM produces a variety of photogrammetric products, from sparse and dense point clouds, to mesh reconstructions and orthomosaics. The software relies on several open source libraries, including OpenSfM (an SFM library on top of OpenCV), OpenMVS, and CMVS/PMVS2 (or simply CMVS) 6, 9, 17, 19, 48, ODM provides an affordable solution for 

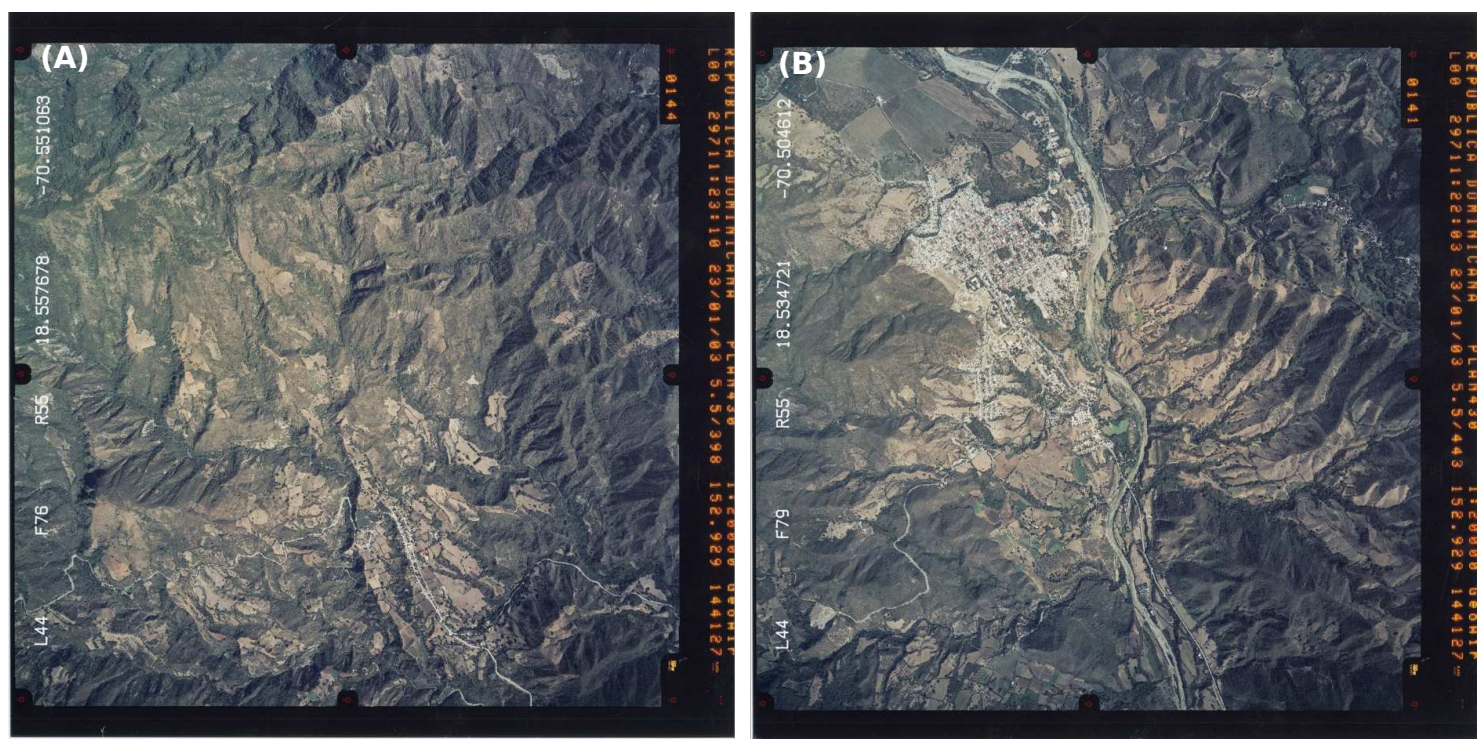

Figure 1. Sample photos from "INDRHI flight", both taken in January 2003. (A) Photo ID L44-F76-R55 showing town of El Pinar (bottom right quadrant) and its surrounding mountains. (B) Photo ID L44-F79-R55, showing town of Ocoa (upper left quadrant) and the river of the same name from top to bottom of the photo 29 .

processing, not only images taken with digital still cameras, but also archived film-based aerial photos.

A recent research demonstrated the suitability of using SFM and MVS commercial software in the production of photogrammetric products from archived aerial photos, proving that this is an efficient way to process large projects in a short time and with reduced costs [22]. In developing countries, and especially in government agencies and academic institutions, proprietary software is often unaffordable and difficult to maintain. The aim of this study was to test the ability of ODM and other open-source software to generate accurate orthomosaics, point clouds, and digital surface models from historical aerial photos.

\section{Materials and Methods}

\subsection{Overview of typical workflow with OpenDroneMap}

ODM performs a complete workflow by prompting other libraries to run specific tasks, mainly OpenSfM and CMVS [17,19]. The typical workflow for generating photogrammetric products from digital images is described below (see Figure 2).

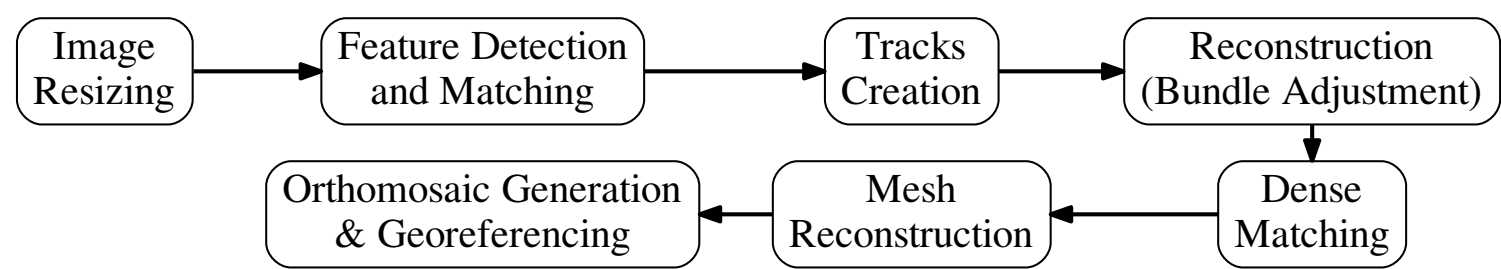

Figure 2. Typical workflow with OpenDroneMap.

Image resizing Because OpenSfM can efficiently reconstruct a sparse point cloud with lowresolution images, the original files can be resized proportionally based on their largest side. This is 
Feature detection and matching OpenSfM retrieves and matches conjugate points between overlapping images by means of the histograms of oriented gradients (HOG) and the Hessian affine feature point detector 14, 18,34,35. By default, OpenSfM detects conjugate points in areas covered by a minimum of three images. This makes sense for images taken by UASs, which usually overlap by $80 \%$. Nevertheless, archived aerial photos typically overlap by $60 \%$ longitudinally and $20-30 \%$ transversally, which means extensive areas are covered by only two images. In order to ensure that OpenSfM reconstructs areas covered by only two photos, a special configuration must be used before starting the feature detection.

Track creation In this step, OpenSfM creates tracks, which are feature points corresponding to the same matching physical points in different images. The algorithm evaluates the list of tracks in order to keep only those found in a preset number of images.

Reconstruction Using the Ceres Solver [2], OpenSfM starts an incremental reconstruction, which can also be called bundle adjustment. This step has the goal of obtaining the relative orientation of the images. In other words, OpenSfM tries to simultaneously determine the 3D positions of the tracks (the sparse point cloud) and the positions of the cameras.

Dense matching This key step has the goal of generating a dense point cloud using an MVS matching algorithm. The user may choose between two alternatives: OpenMVS (bundled with OpenSfM, the default library for dense matching) 9, 48 or CMVS [17. The results and processing time of the workflow vary significantly depending on the algorithm selected. Usually, CMVS is more time consuming than OpenSfM, but the former may produce suitable results, depending on the end-user's intended applications. Both algorithms are described below:

- OpenMVS is based on a multiple view stereo technique, and takes both the accuracy and efficiency into account 9 , 48. For each input image, the algorithm selects a reference image and creates a stereo pair, following the criterion of a minimal baseline. Afterward, depth-maps are computed for selected pixels in the target image by matching them with the corresponding pixels in the reference image at the lowest possible aggregated cost. Because some noise is generated during the process, a refinement is performed by checking the consistency with neighboring depth-maps. Finally, the refined depth-maps are merged together into a single point cloud, avoiding redundancies by applying a neighboring depth-maps test.

- CMVS is an MVS algorithm designed to efficiently generate an accurate dense point cloud 17]. CMVS performs well with a large number of images and few computational resources by decomposing the project into clusters of suitable size. The algorithm is patch-based, which means that it considers a patch as a tangent plane approximation of the actual surface. The patch reconstruction begins with feature detection and feature matching, using the differenceof-Gaussian (DoG) and Harris operators. A patch expansion procedure is repeatedly performed from a set of matched features, using visibility preset parameters to decide the exclusion of false matches. Afterward, a pairwise photometric discrepancy function between a pair of images is determined for each patch. The patches with the lowest discrepancy are recovered and reconstructed, first by initializing their corresponding parameters, and then by optimizing their geometric component, which implies the minimization of their photometric discrepancy score. Finally, three filters based on the visibility, depth-map test, and a weak form of regularization are used to remove erroneous patches.

Mesh reconstruction Both dense matching libraries, OpenSfM and CMVS, generate meshes and textured meshes using the Poisson Surface Reconstruction software 30. Although these products are not usually exploited in the GIS environment, commercial and open-source packages are increasingly 
providing tools for the 3D browsing and editing of meshes, but there is still a long way to go. Alternatively, MeshLab is an advanced 3D open-source cross-platform software package that is suitable for processing the meshes generated by ODM [11].

Orthomosaic generation and georeferencing In this final step, ODM generates an orthomosaic in the PNG format, which is a geometrically corrected image of the entire project area. Furthermore, ODM georeferences both the point cloud and orthomosaic, either by using the GPS coordinates that may be stored in the Exif metadata of the images, or by parsing a GCP file provided by the user. For this final task, ODM relies on both the Geospatial Data Abstraction Library (GDAL) and the Point Data Abstraction Library (PDAL) [8,20].

\subsection{Proposed workflow with OpenDroneMap using historical aerial pho- tos}

Next the proposed workflow using ODM and other open-source software is described, with the goal of preparing and processing historical aerial photos (see Figure 3 ).

88

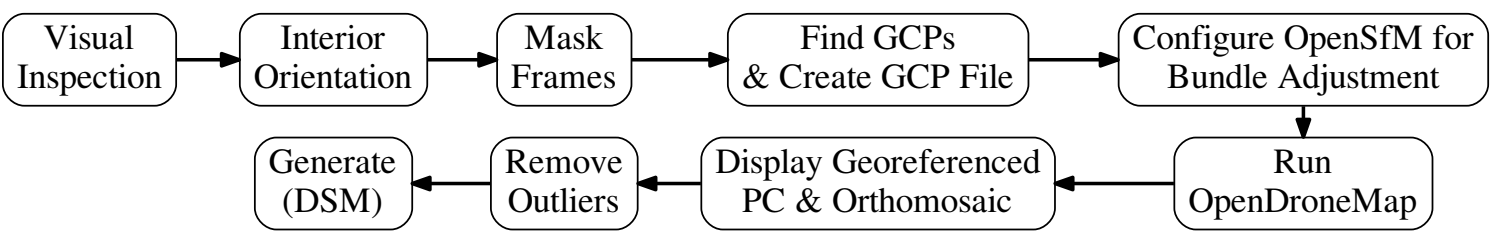

Figure 3. Proposed workflow with OpenDroneMap using archived aerial photos.

Visual inspection Usually, photogrammetric surveys comprise photos taken with different cameras and on different dates. A visual inspection is recommended to determine whether to process all the images simultaneously, or to split the project into separate workflows.

Interior orientation OpenSfM was designed to process images taken by consumer digital cameras for which a known coordinate system exists. Because this is not the case for archived aerial photos, an interior orientation is recommended in order to recover the reference coordinate system of the photos before the reconstruction. Two different scenarios may be considered in this step: when the fiducial marks are visible and camera frame dimensions are known, or when either the fiducial marks are absent or the camera frame dimensions are unknown.

In the first scenario, where the fiducial marks of the photos are available and the dimensions of the camera frame are known, an affine transformation with four points can be used to orient the images. In addition, cropping and resampling operations may be necessary in order to generate images of equal dimensions and with the principal point falling into the center of the image matrix. These steps can be accomplished using either E-foto, QGIS, SAGA, GIMP, GDAL, ImageJ, or ImageMagick, which provide a suitable GUI to assist the user in the collection of image coordinates $13,20,21,28,39,41,47]$. In this research, the QGIS Georeferencer Plugin was selected to collect the source points. In addition, the affine transformation and the cropping and resampling procedures were performed with ImageMagick.

In the second scenario, where the fiducial marks are unavailable or the frame dimensions are unknown, the interior orientation may be performed to register all of the images to an arbitrary selected one within the project 22].

Mask frames Archived aerial photos are usually enclosed in a black box, which represents the frame of the camera. In order to avoid undesirable results during the reconstruction step, the frame may be masked. This can be done using image manipulation software, e.g., GIMP, by selecting the frame area with a tool such as the "intelligent scissors" or "fuzzy select." The area selected is then 
replaced with a black fill, turning it into a mask. Finally, a PNG file containing the mask may be applied to the photos in batch-mode using ImageMagick software.

Find GCPs and create GCP file In order to obtain georeferenced orthomosaics and point clouds, which are suitable for GIS applications, ground control points must be provided to the software. Most of the UASs used in photogrammetric surveys are equipped with several instruments that store the orientation and camera poses. These may include a global navigation satellite system receiver (usually a GPS receiver), an inertial measurement unit (IMU), a compass, and a barometric pressure sensor. The auto-pilot software of the UAS collects and stores the data in the Exif metadata of the image files [4]. ODM, specifically OpenSfM, is designed to exploit these data to accurately perform the SFM algorithm. Nevertheless, when using archived photos, sensors data are absent or difficult to exploit. Therefore, as an alternative, ground control points (GCP) may be provided by the user. These GCPs must be evenly distributed over the entire area, and should be placed over high contrast objects that are visible in at least three images. The GCP file is a list of points stored as simple text, which can be created with E-foto or QGIS. ODM expects a GCP file with the format described as follows. The first line must contain the name of the coordinate reference system in any format readable by the GDAL library 20. The rest of the file should contain the actual GCPs, with each line corresponding to an occurrence of a GCP in one image and comprising the following fields separated by spaces: the $X, Y$, and $Z$ coordinates in the specified reference system of the GCPs; the associated $x$ and $y$ pixel image position; and the corresponding image file name where the GCP appears 50 .

Configure OpenSfM for bundle adjustment OpenSfM was designed for images with large overlap percentages, usually $80 \%$ or greater. Because archived aerial photos have lower overlap percentages, the minimum number of consistent views needed to reconstruct a valid point is two. In addition, it is necessary to configure the depth-map method, which by default is "patch match" but must be set to "brute force," in order to force matching by all the descriptors available and not only based on a distance search [4, 10]. With these options properly set, the number of matched features increased, and a better reconstruction could be obtained.

Run ODM This step was executed by selecting both of the available dense matching algorithms, OpenMVS and CMVS. It is noteworthy that, in order to generate a comprehensive orthomosaic, CMVS was instructed to keep unseen faces in the mesh and to skip the geometric visibility test. In addition, the minimum number of features to extract per image was set to 30,000.

Display georeferenced point cloud and orthomosaic ODM warps both the georeferenced point cloud and the orthomosaic in the same coordinate reference system declared in the GCP file. ODM exports the point clouds into several formats, which include the PLY format (polygon file format, also known as the Stanford triangle format), the LAS format, and the CSV format in an $X Y Z$ layout 5, 33, 46]. The orthomosaic is delivered in the GeoTIFF format. Both the GeoTIFF orthomosaic and CSV point cloud can be displayed in QGIS. Furthermore, using the GRASS GIS tools within QGIS, the LAS file may be imported into a vector map 37. MeshLab may be used to display the PLY file by importing it into a new project. It is important to spot outliers in the point cloud in order to keep track of them in the subsequent removal step.

Remove outliers Typically, the dense matching algorithms generate sparse outliers, which could affect the accuracy of the DSM generated in the subsequent step. Outliers may be removed with either GRASS GIS or with the point cloud library (PCL) [37,45]. Experiments conducted in this research showed that the PCL algorithm, which is called the pcl_outlier_removal, performed efficiently and accurate. Users may choose between two methods, either by establishing a sphere of radius $r$ that searches the $k$-nearest neighbors, or by setting a maximum standard deviation of the
128 129 130 131 132 133 134 135 136 137 138 139 140 141 142 143 144 
$Z$-value from the $k$-nearest neighbors. The latter method was found to perform better than the former, removing the points considered to be outliers and preserving the integrity of the valid ones.

Generate digital surface model (DSM) Several open source packages are available for DSM generation, including GRASS GIS, PCL, SAGA GIS, and GDAL. GRASS GIS performed efficiently and produced an accurate DSM. The algorithm executed was a surface generator that used a bicubic or bilinear spline interpolator with Tykhonov regularization [7].

\section{Results}

The workflow was applied to 10 photos belonging to the strip designated as " $44 \mathrm{~W}$ " of the INDRHI flight, which covers an area of approximately 100 square kilometers of the Ocoa River system (see Figure 4). The area comprises various landforms, mainly slopes, river valleys, and ridges, with altitudes ranging from $300 \mathrm{~m}$ to $1600 \mathrm{~m}$. Achieving high accuracy in a rough topography is a challenging task that is highly dependent on the preparation of the photos and the strategy adopted for GCP searching. The total execution time of the workflow for one person (including the computation time) was $4 \mathrm{~h}$.

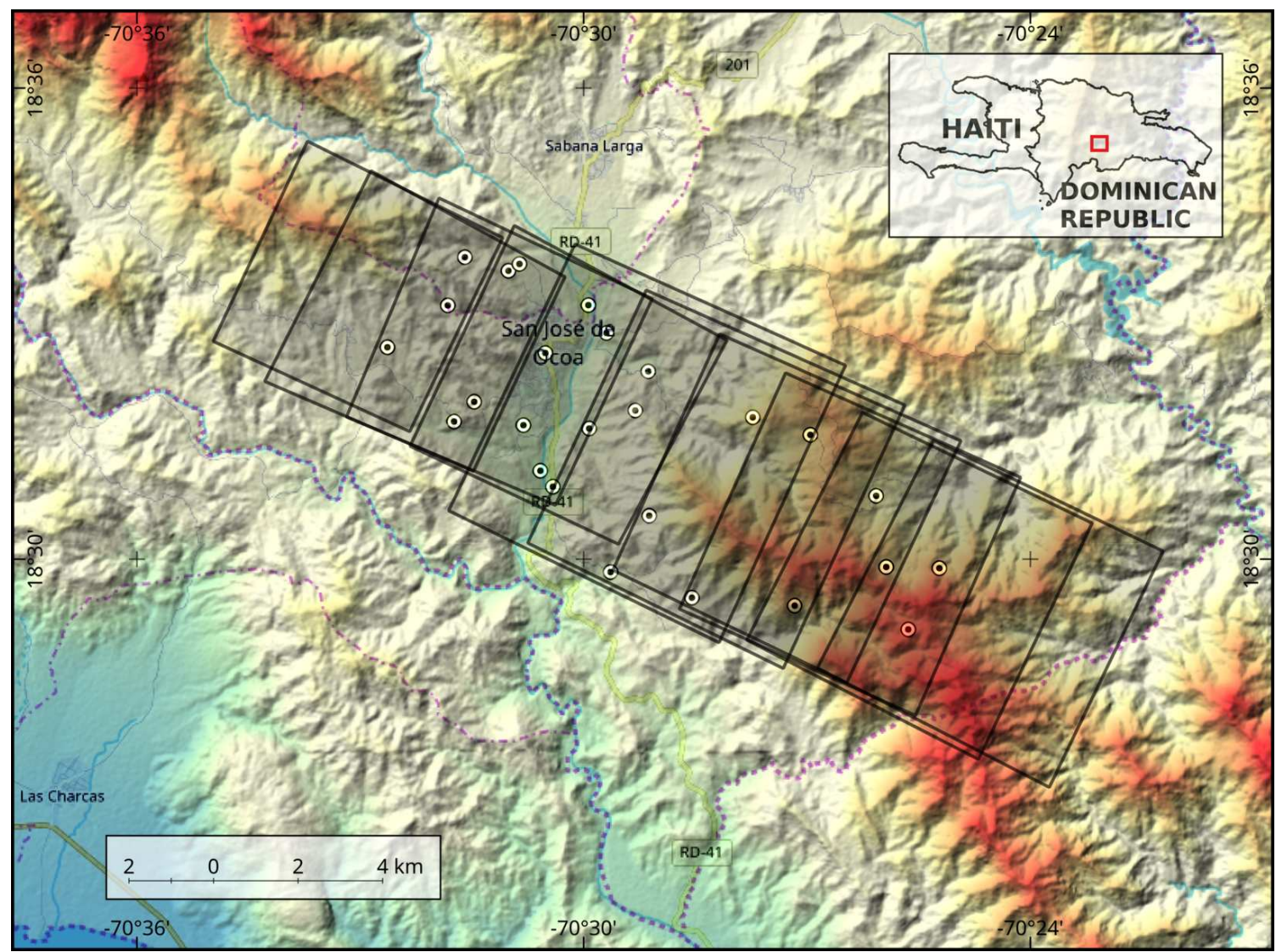

Figure 4. Footprints (shaded polygons) of 10 archived aerial photos of Ocoa River system. The GCPs used for the exterior orientation are depicted as white circles with an inner black dot. Background: color shaded relief from 30-m SRTM DEM (red-white is highland, blue-green is lowland) blended with OpenStreetMap standard layers [36 38$]$. 


\subsection{Image preparation, GCP search and reconstruction}

The interior orientation of the photos was performed with an affine transformation that fitted the images into a frame of $226.5 \mathrm{~mm}$. The photos were cropped up to the extent of the fiducial marks. In addition, the enclosing black boxes of the photos were conveniently masked. In order to support the orientation performed by OpenSfM, the focal distance printed in the photos $(152.929 \mathrm{~mm})$ was passed as a run-time parameter to ODM.

Twenty-six GCPs were used to perform the exterior orientation (see Figure 4). The GCP search strategy focused on both finding features visible in at least three images and achieving good agreement between the georeferenced products and the sources of the GCP coordinates. The $X Y$ coordinates of the GCP were collected from Google Earth services 23, and the corresponding $Z$-values of the GCP were recovered from the topographic maps of the DR [16]. The bundle adjustment performed by OpenSfM obtained both 30,682 conjugate points and 10 camera poses, which are depicted in Figure 5. The next section describes the outcomes generated in the dense matching step using the reconstruction.

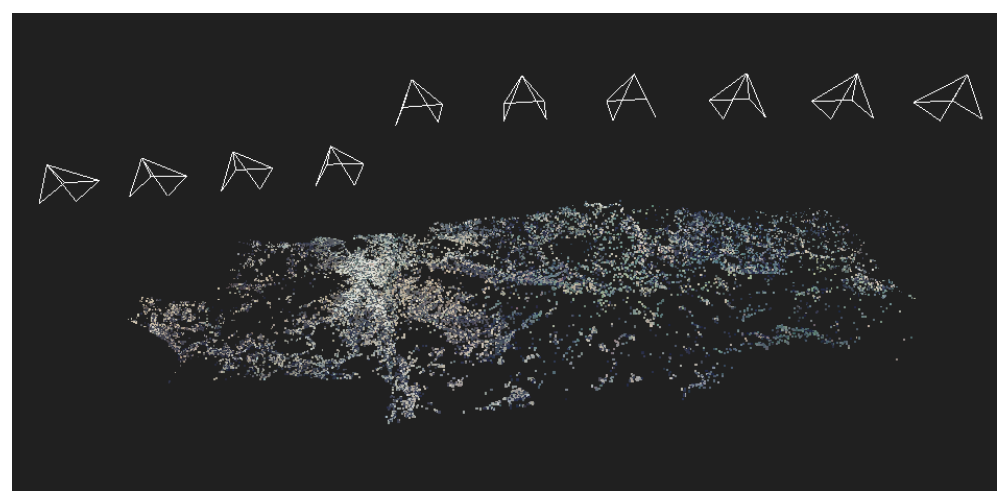

Figure 5. Representation of camera poses and sparse point cloud using OpenSfM reconstruction viewer.

\subsection{Dense matching, DSM generation, and orthomosaics}

Using the reconstruction as an input, the dense matching may be accomplished with either the OpenMVS or CMVS algorithms, which are subsequently used for the generation of DSMs. In addition, each algorithm produces an orthomosaic based on the mesh reconstructed in the previous steps. These outcomes are described next.

OpenMVS generated a point cloud of 1.5 million points. A density estimation using a uniform kernel function yielded a mean density of approximately 12,000 points $/ \mathrm{km}^{2}$. In areas with features visible in at least three images, a maximum of 24,400 points $/ \mathrm{km}^{2}$ was reached. The PCL outlier removal tool discarded 5910 points. A point was deemed to be an outlier if its $Z$-value exceeded by two standard deviations the mean $Z$-value of the 50 nearest neighbors.

The CMVS algorithm produced a point cloud of 3.85 million points, with a density of roughly 31,200 points $/ \mathrm{km}^{2}$. Almost 150,000 points were deemed outliers, and were subsequently removed from the original point cloud, most of them located in the neighboring areas of the frames of the photos.

The DSMs generated with each point cloud are shown in Figure 6. For this task, a spline interpolator with Tykhonov regularization from GRASS GIS was used. In order to avoid excessive smoothing, the Tykhonov regularization parameter was set to 0.01 for both point clouds.

Using the point cloud generated by OpenMVS as an input, the tool computed the mean distance between points, which yielded a value of $9 \mathrm{~m}$. The length of each spline step in both the EW and NS directions was set to $18 \mathrm{~m}$, according to the recommendation of using a value twice the mean distance between points 24. Finally, the resolution of the output raster was set to $2 \mathrm{~m}$. The 

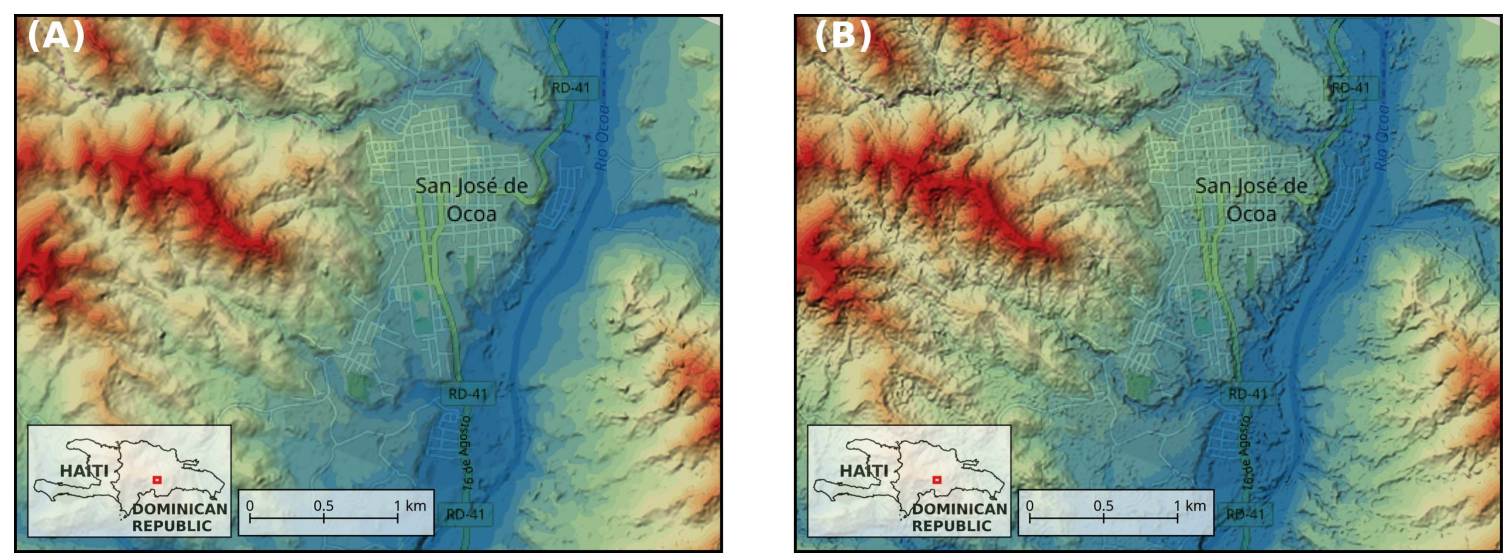

Figure 6. Sample area of color shaded DSMs (red-white is highland, blue-green is lowland) generated from dense point clouds produced by both algorithms: (A) OpenMVS and (B) CMVS. The roughness is greater in the DSM generated by CMVS than in the OpenMVS one. Background: OpenStreetMap standard layers 38 . See text for details.

result is shown in Figure 6A. It is noteworthy that the surfaces of the slopes and river valleys are conspicuously smooth in this DSM. Nevertheless, fluvial banks and terraces are seen as undulating surfaces.

Using the point cloud generated by CMVS as an input, the computed mean distance between points was $6 \mathrm{~m}$. Thus, the length of each spline step for the DSM generation was set to $12 \mathrm{~m}$ (see Figure 6B). This DSM result was coarser than the OpenMVS one (see Figure 6A), especially in vegetated areas. However, a morphological filter and denoising algorithm were tested, which successfully smoothed most of the surfaces, while preserving sharp features [45, 49]. In addition, fluvial terraces were correctly depicted as flat surfaces. Therefore, this DSM may be suitable for geomorphological applications.


Figure 7. Sample areas of orthomosaics produced by both algorithms: (A) OpenMVS and (B) CMVS. The red ellipse encloses artifacts in the seam line of the orthomosaic produced by CMVS.

Finally, both algorithms, OpenMVS and CMVS, generated complete orthomosaics of the area of interest. OpenMVS produced a seamless orthomosaic (Figure $7 \mathrm{~A}$ ), but the one generated by CMVS showed a few artifacts in the seam lines (red ellipse in Figure 7B). A visual inspection showed a good fit between the orthomosaics and Google Earth imagery. 


\subsection{Accuracy assessment of DSMs}

The accuracy assessments of the DSMs generated with both algorithms, OpenMVS and CMVS, are presented in this section. In order to accomplish this task, 55 check points were placed randomly over the area of interest. For each point, the $Z$-values were obtained from both the topomap and DSM. Using these values, the root mean square error (RMSE) of each DSM was calculated. In addition, the null hypothesis of a zero mean difference between the estimated and reference values was tested using a paired $t$-test.

The RMSE of the DSM generated by CMVS was approximately $9 \mathrm{~m}$, and no significant difference was found between the estimated and reference values $(p>0.05)$. For the DSM generated by OpenMVS, the RMSE was $13 \mathrm{~m}$, and significant difference was observed between the estimated and reference heights $(p<0.05)$.

\section{Discussion and perspectives}

This paper showed that the proposed photogrammetric workflow using open-source software packages is suitable for processing historical aerial photos. The workflow was tested using 10 photos of an area of rough topography. The accuracy assessments suggested that the DSMs and the orthomosaics showed good agreement with the reference data.

Other software packages for processing historical aerial photos are available in the open-source community, including MicMac and E-foto 12,44. MicMac offers several advantages, which include a GUI, a well documented step-by-step guide, and an active forum that has a number of discussions of software-related issues and provide photogrammetry reference materials $[40$. E-foto is bundled with a suitable GUI, has good reference materials [15, 39, 42], and a user-support forum exists Nevertheless, the forum showed little activity at the present time.

The workflow proposed in this paper has many advantages, with the main ones being that it relies on third-party software packages which are periodically updated, and the ability for producing point-clouds with two different dense matching algorithms. In addition, the software packages used in the workflow relies on an active open-source community, which offers suitable and quick support. Another advantage is that the algorithms used in the workflow can be tuned by the users in order to get suitable results.

The main drawback of the proposed workflow is the lack of a semi-automatic toolchain. Although each step of the workflow was performed using the command line interpreter, the commands should be executed sequentially by the end-user in order to reproduce the desired results. One solution that will be explored in the near future, is the implementation of a toolchain in Python programming language for allowing users to perform the entire workflow in a semi-automatic way. Another improvement that will be explored is the implementation of an algorithm for searching GCPs and fiducial marks, which may speed-up both interior and exterior orientations steps.

\section{Conclusions}

The proposed photogrammetric workflow with OpenDroneMap and other open-source software was applied to historical aerial photos, which included the preparation of images, selection of GCPs, reconstruction, dense matching with two different algorithms (OpenMVS and CMVS), and the production of DSMs and orthomosaics. The DSM generated from the OpenMVS point cloud was notably smooth, although flat plains were seen as undulating surfaces. The DSM generated from the CMVS point cloud was coarser, and flat surfaces were depicted correctly, making it suitable for geomorphological applications. In addition, both algorithms generated complete orthomosaics, but the one produced by OpenMVS was truly seamless. Accuracy assessments suggested that the proposed workflow may be applicable to other archived photo surveys with high efficiency and at zero cost. 


\section{References}

1. M. Abdel-Wahab, K. Wenzel, and D. Fritsch. Efficient reconstruction of large unordered image datasets for high accuracy photogrammetric applications. In ISPRS Annals of the Photogrammetry, Remote Sensing and Spatial Information Sciences, Melbourne, Australia. XXII ISPRS Congress, volume 3, pages 1-6, 2012.

2. S. Agarwal, K. Mierle, and Others. Ceres solver. http://ceres-solver.org, 2018.

3. D. Benjamin, S. Fitzsimmons, and P. Toffanin. OpenDroneMap, 2018. https://github. com/OpenDroneMap and http://opendronemap.org/. [Online; accessed May-2018].

4. E. Bostanci, N. Kanwal, B. Bostanci, and M. S. Guzel. A fuzzy brute force matching method for binary image features. arXiv preprint arXiv:1704.06018, 2017.

5. P. Bourke. Data Formats: 3D, Audio, Image, 2018. [Online; accessed May-2018].

6. G. Bradski. The OpenCV Library. Dr. Dobb's Journal of Software Tools, 2000.

7. M. A. Brovelli, M. Cannata, and U. M. Longoni. LIDAR data filtering and DTM interpolation within GRASS. Transactions in GIS, 8(2):155-174, 2004.

8. H. Butler, M. Gerlek, A. Bell, P. Ramsey, B. Chambers, M. Loskot, C. Foster, P. Gadomski, and K. McKelvey. PDAL - Point Data Abstraction Library, 2015. http://www.pdal.io/ index.html and https://github.com/PDAL/PDAL. [Online; accessed May-2018].

9. D. Cemea. OpenMVS, 2015. [Online; accessed May-2018].

10. J. Cheng, C. Leng, J. Wu, H. Cui, H. Lu, et al. Fast and accurate image matching with cascade hashing for 3d reconstruction. In 2014 IEEE Conf. on Computer Vision and Pattern Recognition (CVPR), pages 1-8. IEEE, 2014.

11. P. Cignoni, M. Callieri, M. Corsini, M. Dellepiane, F. Ganovelli, and G. Ranzuglia. Meshlab: an open-source mesh processing tool. In Eurographics Italian Chapter Conference, volume 2008, pages 129-136, 2008.

12. L. Coelho and J. N. Brito. Fotogrametria digital. EdUERJ, 2007.

13. O. Conrad, B. Bechtel, M. Bock, H. Dietrich, E. Fischer, L. Gerlitz, J. Wehberg, V. Wichmann, and J. Böhner. System for automated geoscientific analyses (SAGA) v.2.1.4. Geoscientific Model Development, 8(7):1991, 2015.

14. N. Dalal and B. Triggs. Histograms of oriented gradients for human detection. In Computer Vision and Pattern Recognition, 2005. CVPR 2005. IEEE Computer Society Conference on, volume 1, pages 886-893. IEEE, 2005.

15. De Souza e Simões Figueiredo, Lia and Dacome Lima, Rodrigo. The E-Foto project, 2016.

16. DMA/IGU. Dominican Republic 1:50000 (Mapa Topográfico Escala 1:50000 de República Dominicana), serie E733, Edición 2, 1962-1968.

17. Y. Furukawa and J. Ponce. Accurate, dense, and robust multi-view stereopsis. IEEE Trans. on Pattern Analysis and Machine Intelligence, 32(8):1362-1376, 2010.

18. P. Gargallo. Using opensfm, 2018. [Online; accessed May-2018].

19. P. Gargallo, Y. Kuang, et al. Opensfm, 2018. [Online; accessed May-2018].

20. GDAL Development Team. GDAL - Geospatial Data Abstraction Library, Version 2.3.0. Open Source Geospatial Foundation, 2018. 
21. GNOME Foundation, P. Mattis, and S. Kimball. GIMP. GNU Image Manipulation Program, 2018. [Online; accessed May-2018].

22. J. Gonçalves. Automatic orientation and mosaicking of archived aerial photography using structure from motion. The International Archives of Photogrammetry, Remote Sensing and Spatial Information Sciences, 40:123, 2016.

23. Google. Google Earth, 2018. [Online; accessed May-2018].

24. E. Hardin, H. Mitasova, L. Tateosian, and M. Overton. GIS-based analysis of coastal Lidar time-series. Springer, 2014.

25. IGU. Fotografías aéreas verticales, Proyecto ICM-58, 1958.

26. IGU. Fotografías aéreas verticales, Proyecto DRA/DRB, 1967.

27. IGU. Fotografías aéreas verticales, Proyecto Manejo de Recursos Naturales (MARENA), 1984.

28. ImageMagick Studio LLC. ImageMagick, 2018. https://www .imagemagick.org/and https: //github.com/ImageMagick/ImageMagick. [Online; accessed May-2018].

29. INDRHI. Photogrammetric aerial survey of the Dominican Republic, 1:20000 scale color photos ("INDRHI flight"), 2000-2003.

30. M. Kazhdan, M. Bolitho, and H. Hoppe. Poisson surface reconstruction. In Proceedings of the Fourth Eurographics Symposium on Geometry Processing, SGP '06, pages 61-70, Aire-la-Ville, Switzerland, Switzerland, 2006. Eurographics Association.

31. U. Lieberwirth, M. Metz, M. Neteler, and K. Kühnle. Applying low budget equipment and open source software for high resolution documentation of archaeological stratigraphy and features. Across Space and Time, page 104, 2013.

32. Mapillary. Mapillary, 2018. [Online; accessed May-2018].

33. K. McHenry and P. Bajcsy. An overview of 3d data content, file formats and viewers. National Center for Supercomputing Applications, 1205:22, 2008.

34. K. Mikolajczyk and C. Schmid. Scale \& affine invariant interest point detectors. International journal of computer vision, 60(1):63-86, 2004.

35. K. Mikolajczyk, T. Tuytelaars, C. Schmid, A. Zisserman, J. Matas, F. Schaffalitzky, T. Kadir, and L. Van Gool. A comparison of affine region detectors. International journal of computer vision, 65(1-2):43-72, 2005.

36. NASA LP DAAC. SRTM 1 Arc-Second Global. https://earthexplorer.usgs.gov/, 2000. published September 2014, accessed May 2016.

37. M. Neteler and H. Mitasova. Open source GIS: a GRASS GIS approach, volume 689. Springer Science \& Business Media, 2013.

38. OpenStreetMap contributors. Planet dump retrieved from https://planet.osm.org . https: //www.openstreetmap.org, 2017.

39. Photogrammetry Lab of the Rio de Janeiro State University's School of Engineering. E-foto. A free GNU/GPL educational digital photogrammetric workstation, 2016. [Online; accessed May-2018].

40. M. Pierrot-Deseilligny, D. Jouin, J. Belvaux, G. Maillet, L. Girod, E. Rupnik, J. Muller, M. Daakir, G. Choqueux, and M. Deveau. Micmac, apero, pastis and other beverages in a nutshell. Institut Géographique National, 2014. 
41. QGIS Development Team. QGIS. A Free and Open Source Geographic Information System, 2018. [Online; accessed May-2018].

42. J. A. Ribeiro, M. Pierrot-Deseilligny, O. B. F. Brito, and G. L. Abelha. E-foto and micmac: synergetic benefits of integrating open-source digital photogrammetry software. In Proceedings of the 3rd Open Source Geospatial ResearchES Education Symposium, page 47, 2014.

43. M. Rumpler, S. Daftry, A. Tscharf, R. Prettenthaler, C. Hoppe, G. Mayer, and H. Bischof. Automated end-to-end workflow for precise and geo-accurate reconstructions using fiducial markers. ISPRS Annals of the Photogrammetry, Remote Sensing and Spatial Information Sciences, 2(3):135, 2014.

44. E. Rupnik, M. Daakir, and M. P. Deseilligny. MicMac-a free, open-source solution for photogrammetry. Open Geospatial Data, Software and Standards, 2(1):14, 2017.

45. R. B. Rusu and S. Cousins. 3D is here: Point Cloud Library (PCL). In IEEE International Conference on Robotics and Automation (ICRA), Shanghai, China, May 9-132011.

46. A. Samberg. An implementation of the ASPRS LAS standard. In ISPRS Workshop on Laser Scanning and SilviLaser, pages 363-372, 2007.

47. C. A. Schneider, W. S. Rasband, and K. W. Eliceiri. NIH Image to ImageJ: 25 years of image analysis. Nature methods, 9(7):671, 2012.

48. S. Shen. Accurate multiple view 3d reconstruction using patch-based stereo for large-scale scenes. IEEE transactions on image processing, 22(5):1901-1914, 2013.

49. X. Sun, P. Rosin, R. Martin, and F. Langbein. Fast and effective feature-preserving mesh denoising. IEEE transactions on visualization and computer graphics, 13(5), 2007.

50. P. Toffanin. Running OpenDroneMap, 2018. [Online; accessed May-2018]. 